\title{
Increased expression of T cell immunoglobulin and mucin domain 3 aggravates brain inflammation via regulation of the function of microglia/macrophages after intracerebral hemorrhage in mice
}

\author{
ChangJun Xu, Tao Wang, Si Cheng and YuGuang Liu*
}

\begin{abstract}
Background: Microglia/macrophages are known to play important roles in initiating brain inflammation after spontaneous intracerebral hemorrhage $(\mathrm{ICH})$. T cell immunoglobulin and mucin domain-3 (Tim-3) have been proven to play a critical part in several inflammatory diseases through regulation of both adaptive and innate immune responses. Tim-3 can be expressed by microglia/macrophages and regulates their function in the innate immune response. However, the effect of Tim-3 on inflammatory responses following ICH is unclear.

Methods: In this study, we investigated Tim-3 expression, the inflammatory cytokines tumor necrosis factor-a (TNF- $\alpha$ ) and interleukin-1 $\beta(\mathrm{IL}-1 \beta)$, and brain water content in peri-hematomal brain tissue at 12 hours and at 1, 3, 5, and 7 days post-ICH in wild type (WT) ICH and Tim- $3^{-1-} \mathrm{ICH}$ mice. The numbers of Tim-3 positive cells,astrocytes, neutrophils and microglia/macrophages were detected using immunofluorescence staining. Cytokines were measured by ELISA. Double immunoflurorescence labeling was performed to identify the cellular source of Tim-3 expression. Mouse neurological deficit scores were assessed through animal behavior.

Results: Expression of Tim-3 increased early in mouse peri-hematomal brain tissue after autologous blood injection, peaked at day 1, and was positively correlated with the concentrations of TNF-a, IL-1 $\beta$, and brain water content. Tim-3 was predominantly expressed in microglia/macrophages. Compared with WT mice, Tim- $3^{-1-}$ mice had reduced ICH-induced brain inflammation with decreased TNF- $a$ and IL-1 $\beta$, cerebral edema and neurological deficit scores. Moreover, Tim-/- inhibited activation of microglia/macrophages. The number of activated microglia/ macrophages in $\mathrm{Tim}_{-3^{-/-}} \mathrm{ICH}$ mice was much lower than that in WT ICH mice.

Conclusions: Our findings demonstrate that Tim-3 plays an important role in brain inflammation after $\mathrm{ICH}$, and may be a potential treatment target.
\end{abstract}

Keywords: T cell immunoglobulin, Mucin domain 3, Brain inflammation, Microglia, Macrophages, TNF-a, IL-1 $\beta$

\footnotetext{
* Correspondence: liuyuguang3000@163.com

Department of Neurosurgery, Qilu Hospital of Shandong University, No.107

Wenhuaxi road, Jinan, Shandong 250012, PR China
} 


\section{Background}

Spontaneous intracerebral hemorrhage (ICH) is a common disease with high mortality and morbidity, accounting for 15 to $20 \%$ of all stokes and affecting more than 2 million people worldwide each year $[1,2]$. The mortality rate of $\mathrm{ICH}$ is more than $40 \%$ and only $20 \%$ of survivors can live independently within six months [3]. But until now, there has been no satisfactory treatment in clinical practice, mainly because the mechanisms of brain damage after $\mathrm{ICH}$ are unclear. Increasing researches show that inflammatory response plays an important role in ICH-induced secondary brain damage $[4,5]$. Brain inflammation after ICH is characterized by accumulation of activated inflammatory cells, such as blood-derived cells (macrophages, leukocytes) and brain resident cells (astrocytes, microglia and mast cells). These reactive cells can release inflammatory mediators, including chemokines, cytokine, protease, prostaglandins and other immunoactive molecules [6,7]. The microglia are the first cells to react to brain damage among all the inflammatory cells. Activated microglia have a similar shape to the blood-derived macrophages, therefore microglia are also called as the brain macrophages. Increasing evidence indicates that microglia/macrophages are activated early following $\mathrm{ICH}$, and release a series of toxic factors, including chemokines, cytokines, reactive oxygen species (ROS), cyclooxygenase-2, protease, heme oxygenase- 1 and prostaglandins [8-10]. Therefore, microglia/macrophages play important roles in the secondary brain damage. Tim-3 is a new immuno-regulation molecule found in 2002, which is expressed specially in activated Th1 cells $[11,12]$. Moreover, it has been proven that Tim-3 is also expressed in the cells of the innate immune system, including macrophages, mast cells and dendritic cells [13,14]. Tim-3 expression in microglia/macrophages can be upregulated and induce the production of proinflammatory cytokines, such as tumor necrosis factor-a (TNF-a) and interleukin$1 \beta$ (IL-1 $1 \beta$ ), which can aggravate inflammation and secondary brain damage [14]. However, until now, the effect of Tim-3 on inflammatory response following ICH has been unclear. Considering that the microglia/macrophages are the key cells for inducing brain inflammation and secondary brain damage, and that Tim-3 can regulate the function of microglia/macrophages, we hypothesized that Tim-3 possibly took part in ICH-induced inflammation by regulating the function of microglia/macrophages. This experiment was done to prove our hypothesis.

\section{Materials and methods}

\section{Intracerebral hemorrhage model}

The ICH model of mice was established using the method described previously [15]. Animals were anesthetized with chloral hydrate $(40 \mathrm{mg} / \mathrm{kg}$, intraperitoneal injection). A catheter plugged in the right femoral artery was used to monitor continuous blood pressure and to take blood sampling. Mice were fixed in a stereotactic frame (Stoelting, Kiel, WI, USA). The puncture point was located $1 \mathrm{~mm}$ anterior to the bregma and $2.5 \mathrm{~mm}$ lateral to midline. A 1-mm cranial hole was drilled with dental bit and a 27 -gauge needle was inserted stereotaxically into the right basal ganglia (4 $\mathrm{mm}$ deep). Then, $25 \mu \mathrm{L}$ of autologous blood was injected using a microinfusion pump at a rate of $2.5 \mu \mathrm{L} / \mathrm{min}$. After the infusion, the needle was kept in the place for another 10 minutes to prevent blood leakage, and then the needle was pulled out. The cranial hole was sealed with bone wax and the skin was sutured. Control mice were injected with $25 \mu \mathrm{L}$ $0.9 \%$ saline. Then mice were allowed to recover. During operation, rectal temperature of mice was maintained at $37 \pm 1^{\circ} \mathrm{C}$. Mean arterial blood pressure (MABP) $(\mathrm{mmHg})$, arterial $\mathrm{pH}$, arterial $\mathrm{PO}_{2}, \mathrm{PCO}_{2}, \mathrm{Hb}(\mathrm{g} / \mathrm{L})$, and glucose levels $(\mathrm{mg} / \mathrm{dL})$ were monitored and maintained for stability.

\section{Animals and grouping}

C57BL/ 6 mice (male, 8 to 10 weeks old, and weight 20 to $25 \mathrm{~g}$ ) were purchased from the Animal House Center, Medical College of Shangdong University (Jinan, China). Transgenic line Tim- $3^{-/-}$mice (8 to 10 weeks old, weight 20 to $24 \mathrm{~g}$ ) were purchased from American Jackson Laboratories (Bar Harbor, ME, USA) and were backcrossed to C57BL/6 mice more than eight times. All animals were housed in individual cages under a $12 \mathrm{hr} /$ $12 \mathrm{hr}$ light-dark cycle with temperature $21 \pm 1^{\circ} \mathrm{C}$, humidity 50 to $60 \%$ and free access to food and water. All animals care and experimental protocols were approved by theanimal Ethics Committee of the Sandong University. All efforts were made to minimize the animals' pain and to reduce the number of animals. Different experimental groups were included: 1$)$ For the WT sham group $(n=60)$, $25 \mu \mathrm{L}$ of $0.9 \%$ saline was injected into the brain of these mice. 2) For the WT ICH group ( $n=60), 25 \mu \mathrm{L}$ of autologous blood was injected into the right basal ganglia. 3) For the $\mathrm{Tim}-3^{-/-}$sham group $(\mathrm{n}=50)$,the operation was the same as for the WT sham group. 4) For the Tim- $3^{-/-}$ICH group $(n=50)$,the operation was the same as for the WT $\mathrm{ICH}$ group. A total of 8 mice $(5$ mice in the WT ICH group and 3 mice in the Tim- $3^{-/-}$ICH group) died in our experiment, and the number of mice supplemented was the same. There were 228 mice in our experiment.

\section{Tissue preparation}

In the WT sham group and the WT ICH group, 12 mice were anesthetized at each of the following time points: 12 hours and 1, 3, 5, and 7 days after injection. Half of the mice $(n=6)$ were directly decapitated and their brains were obtained to store at $-80^{\circ} \mathrm{C}$ for use inrealtime RT-PCR, in ELISA and to determine brain water 
content. The others $(n=6)$ were used for immunofluorescence. These mice underwent transcardial perfusion with $200 \mathrm{ml}$ of phosphate buffered saline (PBS), followed by $100 \mathrm{ml}$ of $4 \%$ paraformaldehyde (PFA) in $0.1 \mathrm{M}$ PBS as described before [16]. The brains were removed and postfixed for 24 hours in 4\% PFA, and then were placed in $30 \%$ sucrose until sinking. Coronal brain sections of $10 \mu \mathrm{m}$ thickness were obtained with a freezing microtome (Leica, Nussloch, Germany) and were kept at $-20^{\circ} \mathrm{C}$. In the $\mathrm{Tim}-3^{-/-}$sham group and Tim- $3^{-/-}$ICH group, 10 mice were killed at each of the following time points: 12 hours and 1, 3, 5, and 7 days after operation, Among this total, 6 mice were used for real-time RT-PCR, for ELISA and to determine brain water content, and the others $(n=4)$ were used for immunofluorescence.

\section{Immunofluorescence staining and cell counting}

After being washed in PBS for $10 \mathrm{~min}$, the sections were incubated with $5 \%$ bovine serum albumin for 60 minutes in order to block the nonspecific binding, and then were incubated with goat anti-rat Tim-3 primary antibody (1:100; R\&D systems, Minneapolis, MN, USA. http:// www.rndsystems.com/) at $4^{\circ} \mathrm{C}$ all the night. After being washed three times with PBS, the sections were incubated with secondary antibody goat anti-rabbit IgG (1:100; KPL, Maryland, USA. http://www.kpl.com/home. $\mathrm{cfm})$ for 60 minutes at the room's temperature. The sections were rinsed $3 \times 5 \mathrm{~min}$ and were coverslipped with ProLong antifade medium (Molecular Probes, Eugene, OR, USA). The Tim-3 positive cells were visualized using a fluorescent microscope (Olympus BX51, Japan). For each animal, six representative sections of each brain were selected. Tim-3 positive cells were counted blindly in the approximately $40,000 \mu \mathrm{m}^{2}$ of brain tissues around blood clot. In order to further identify the cellular resource of Tim-3 after ICH, double immunofluorescence labeling [9] was performed by simultaneous incubation of goat anti-rat Tim-3 primary antibody with rat antimouse CD11b (1:200, eBioscience San Diego, CA, USA. http://www.ebioscience.com/) as marker of activated microglia/macrophage, or rabbit anti-GFAP (1:200, Invitrogen, Carlsbad, CA, USA. www.invitrogen.com) as marker of astrocyte, or rabbit anti-MPO (1:100, Dako, Denmark. www.dako.com) as mark of neutrophils. In each group, the number of CD11b positive cells (activated microglia/macrophage), GFAP positive cells (astrocyte) and MPO positive cells (neutrophils) were counted using the same method as used for Tim-3 positive cells. Ipp6.0 image processing software was utilized to count the number of Tim-3 positive cells.

Real-time reverse transcription polymerase chain reaction According to the methods described previously [15], frozen mice brains were homogenized, and total RNA was obtained from about $4 \times 4 \times 4 \mathrm{~mm}^{3}$ volume of perihematomal tissues (blood clot as center under a stereomicroscope) at 12 hours and at 1, 3, 5 and 7 days post-ICH using Trizol reagent (Invitrogen, Carlsbad, CA, USA) in compliance with the manufacture's instruction. The character of RNA was tested by a spectrophotometer (DU800, Beckman, Palo Alto, CA, USA). The M-MLV Reverse Transcriptase System (Promega, Madison, WI, USA) was performed for reverse transcription. The cDNA was stored at $-20^{\circ} \mathrm{C}$. Quantitative real-time PCR was fulfilled with a LightCycler (Roche Diagnostics, Mannheim, GM) and with SYBR Green I in SYBR RT-PCR Kit (TaKaRa Biotechnology, Dalian, China) so as to enlarge and detect the expression of Tim-3 mRNA. The transcript amount of the rat $\beta$-actin housekeeping gene was quantified as an internal RNA control. Primers were purchased from BioAsia Corp. (Shanghai, China). The primer sequences were as follows: $\beta$-actin forward: 5'-GGCATCGTGATGGACT CCG -3 ' and $\beta$-actin reverse: 5'-GCTGGAAGGTGGA CAGCGA -3'; Tim-3 forward: 5'ACTGGTGACCCTCC ATAATAACA -3 ' and Tim-3 reverse: 5'ATTTTCCTCA GAGCGAATCCT $-3^{\prime}$. Experiments were carried out in triplicate for each data point. A threshold cycle value (CT) was calculated by the $\Delta \Delta C T$ method as previously described [17]. The data were analyzed by using Light Cycler Software 4.0 (Roche Diagnostics).

\section{Enzyme-linked immunosorbent assay}

IL-1 $\beta$ and TNF-aare two important inflammatory mediators and can represent the severity of brain inflammation after ICH. The concentrations of IL- $1 \beta$ and TNF-a in brain tissues of the peri-hematomal region were detected via the ELISA method according to the manufacturer's instructions (R\&D systems, Minneapolis, MN, USA). Brain tissues were centrifuged at $12000 \mathrm{rps}$ for $20 \mathrm{~min}$ and the supernatant was collected for analysis. The detection threshold of this assay was $<1 \mathrm{pg} / \mathrm{mL}$.

\section{Brain water content}

Brain water content indicates the degree of brain edema, which is mainly due to peri-hematomal inflammation and blood-brain barrier breakdown. Brain samples were immediately weighed on an electric analytical balance to get the wet weight, and then dried at $100^{\circ} \mathrm{C}$ for 24 hours to obtain the dry weight. Brain water content $(\%)=($ wet weight - dry weight)/wet weight of brain tissue $\times 100$.

\section{Testing of neurological deficit scores}

At 12 hours and at 1, 3, 5 and 7 days after operation, the neurofunctional abnormality of mice was tested and scored in each group. An observer blinded to the identity of the mice evaluated behavior. Three behavioral examinations, including ipsilateral circling, forelimb flexion and beam balance were used as described before 
[18]. Each point is graded from 0 to 4 . The maximum abnormal score is 12 .

\section{Statistical analysis}

All statistical analyses were performed with SPSS 16.0 for Windows (Chicago, IL, USA). Data are presented as mean \pm standard deviation (SD). Multiple group differences were analyzed using one-way or two-way analysis of variance and Student-Newman-Keuls test in post hoc tests. An independent-samples t-test was adopted for comparison of the two groups. The correlation analysis was completed by bivariate. $P<0.05$ was considered as the indication of statistical significance.

\section{Results}

The physiological parameters in four groups during production of hematoma

During production of hematoma, we monitored rectal temperature, MABP, arterial $\mathrm{pH}$, arterial $\mathrm{PO}_{2}, \mathrm{PCO}_{2}, \mathrm{Hb}$, and glucose levels in the four groups. The results showed there was no difference among all groups $(P>0.05)$ (Table 1).

\section{Increase of Tim-3 expression in the peri-hematomal brain} tissues

In order to investigate the expression of Tim-3 in the peri-hematomal brain tissues, we observed the number of Tim-3 positive cells in the peri-hematomal brain tissues at 12 hours and at 1, 3, 5 and 7 days post-ICH. Results indicated that the number of Tim-3 positive cells in the WT ICH group began to increase at 12 hours, peaked at Day 1, and decreased at Day 3 after $\mathrm{ICH}$. There was a significant difference when compared with that of WT sham mice in all time-tested points $(P<0.01)$ (Figure 1A,B). Furthermore, we studied Tim-3 mRNA expression in the peri-hematomal brain tissues at 12 hours and at 1, 3, 5 and 7 days post-ICH using a real-time RTPCR method. The change trend of Tim-3 mRNA was similar to the number of Tim-3 positive cells. The Tim-3 mRNA expression was strikingly upregulated in the perihematomal brain tissues after $\mathrm{ICH}$ at 12 hours, peaked at
Day 1, and descended at Day 3. The Tim-3 mRNA expression was significantly different when compared with that in WT sham $\operatorname{group}(P<0.01)$ (Figure $1 C$ ).

\section{Preponderant expression of Tim-3 in microglia/macrophages} To identify the cellular resource of Tim-3 expression, we observed double-immunofluorescence staining. The results revealed that expression of Tim-3 was preponderant in $\mathrm{CD}_{11 b^{+}}$cells (microglia/macrophages), was lower in $\mathrm{MPO}^{+}$cells (neutrophils), and was lowest in $\mathrm{GFAP}^{+}$ cells (astrocytes) (Figure 2).

\section{Obvious increase of IL-1 $\beta$, TNF-a and brain water content} in the peri-hematomal brain tissues

IL-1 $\beta$ and TNF-a are two main proinflammatory cytokines of microglial source, which represent the level of brain inflammation $[19,20]$. We detected the concentration of IL-1 $\beta$ and TNF-a in the peri-hematomal brain tissues at 12 hours and at 1, 3, 5 and 7 days post-ICH. Both of them elevated obviously in the WT ICH group at all tested time points. There was significant difference between the WT ICH group and the WT sham group $(P<0.01)$. IL-1 $\beta$ and TNF-a increased at 12 hours after $\mathrm{ICH}$, and peaked at Day 1 , then decreased at Day 3 (Figure 3A,B). We also measured the mice brain water content to judge the degree of brain edema. Brain water content heightened at 12 hours after ICH, peaked at Day 1 and Day 3, and then gradually declined. There was a significant difference between the WT ICH group and the WT sham group $(P<0.01)$ (Figure $3 C)$.

\section{Positive correlation of expression of Tim-3 with IL-1 $\beta$, TNF-a and brain water content}

We analyzed the correlation between expression of Tim-3 and IL-1 $\beta$, TNF-a and brain water content. The results show that expression of Tim-3 was positively correlated with IL-1 $\beta \quad(r=0.618, \quad P<0.001)$, TNF-a $(r=0.610, P<0.001)$ and brain water content $(r=0.566, P=0.001)$ (Figure $4 \mathrm{~A}, \mathrm{~B}, \mathrm{C})$.

Table 1 Comparison of physiological parameters ${ }^{\mathrm{a}}$ (PP) in all groups during production of hematoma

\begin{tabular}{|c|c|c|c|c|}
\hline & WT sham group $(n=60)$ & WT ICH group $(n=60)$ & Tim $-3^{-/}$sham group $(n=50)$ & Tim $-3^{-1-}$ ICH group $(n=50)$ \\
\hline Rectal temperature & $36.97 \pm 0.54$ & $37.12 \pm 0.42$ & $37.06 \pm 0.49$ & $37.01 \pm 0.39$ \\
\hline $\mathrm{MABP}(\mathrm{mmHg})$ & $100.04 \pm 1.86$ & $101.22 \pm 1.96$ & $99.75 \pm 2.09$ & $100.09 \pm 1.66$ \\
\hline Arterial pH & $7.38 \pm 0.04$ & $7.41 \pm 0.02$ & $7.40 \pm 0.05$ & $7.35 \pm 0.02$ \\
\hline $\mathrm{PO}_{2}(\mathrm{mmHg})$ & $106.57 \pm 8.50$ & $110.21 \pm 5.84$ & $109.86 \pm 9.71$ & $103.34 \pm 5.04$ \\
\hline $\mathrm{PCO}_{2}(\mathrm{mmHg})$ & $42.54 \pm 4.12$ & $41.58 \pm 2.71$ & $41.77 \pm 3.07$ & $42.37 \pm 3.42$ \\
\hline $\mathrm{Hb}(\mathrm{g} / \mathrm{L})$ & $149.17 \pm 6.79$ & $147.01 \pm 6.86$ & $143.49 \pm 8.64$ & $149.06 \pm 7.48$ \\
\hline Glucose (mg/dL) & $135.90 \pm 14.76$ & $137.16 \pm 8.47$ & $134.64 \pm 16.38$ & $137.72 \pm 10.08$ \\
\hline
\end{tabular}

${ }^{a}$ Data were presented as mean \pm standard deviation. The physiological parameters were not differentamong all groups. $P>0.05 . \mathrm{ICH}$, intracerebral hemorrhage; Tim-3, T cell immunoglobulin and mucin domain-3; WT, wild type. 


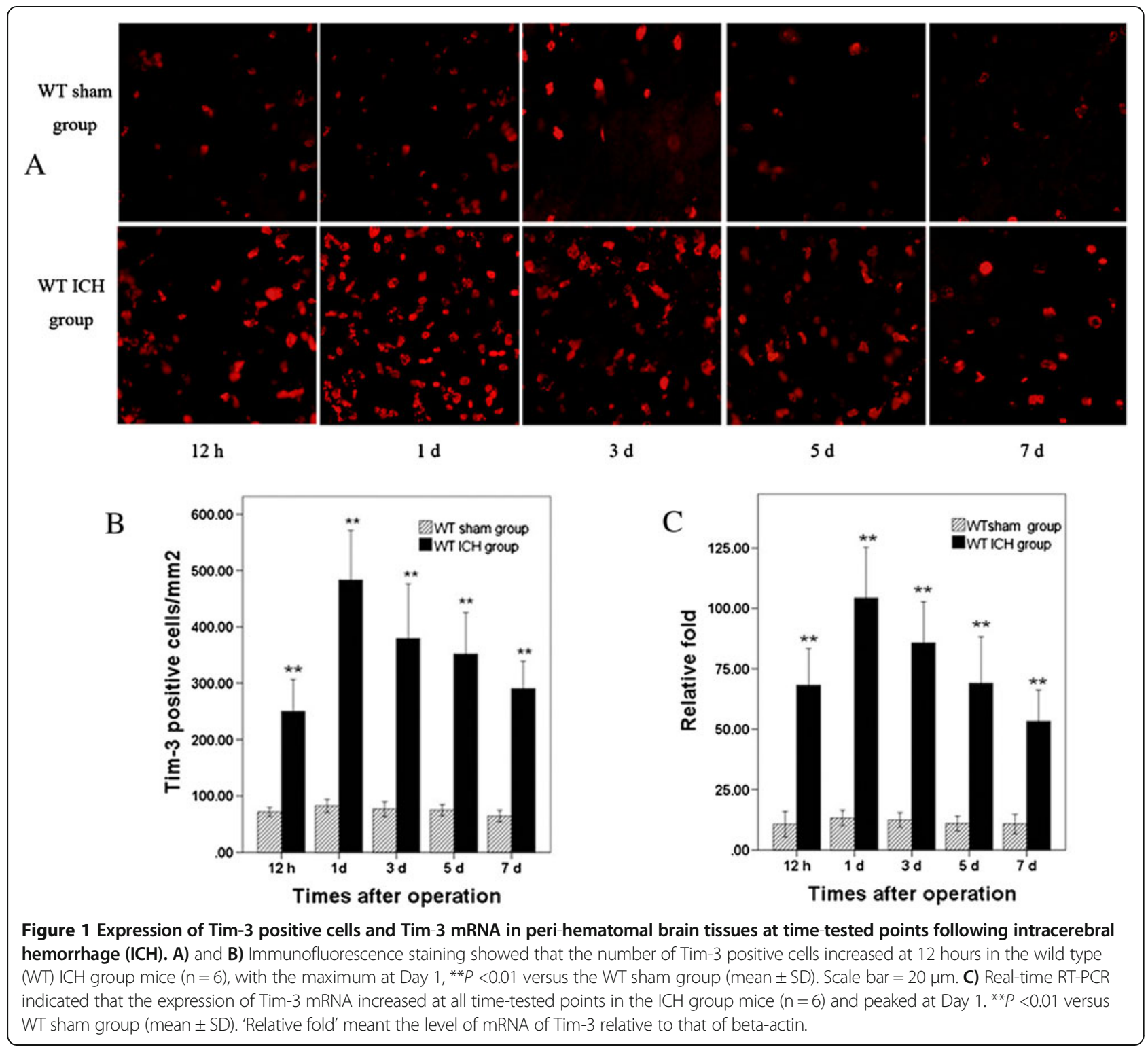

The brain inflammatory response, brain edema and neurologic function in the Tim $-3^{-/-} \mathrm{ICH}$ mice

To further prove the effects of Tim-3 on brain inflammation, brain edema and neurologic function, we compared the concentration of IL-1 $\beta$ and TNF-a, brain water content and NDS in the WT ICH group with that in the Tim $-3^{-1-} \mathrm{ICH}$ group. The concentration of both IL-1 $\beta$ and TNF-a in the peri-hematomal brain tissues notably decreased in the Tim- $3^{-/-} \mathrm{ICH}$ group, and there was significant difference compared to that in the WT ICH group (the $\mathrm{Tim}-3^{-/-} \mathrm{ICH}$ group versus the WT ICH group, IL-1 $\beta$ : 12 h $P<0.01$; $1 \mathrm{~d} P<0.05$; $3 \mathrm{~d} P<0.05$; $5 \mathrm{~d} P<0.05$; 7d $P<0.01$. TNF-a: $12 \mathrm{~h} P<0.05$; $1 \mathrm{~d}$ $P<0.05$; 3d $P<0.01$; $5 \mathrm{~d} P<0.01$; $7 \mathrm{~d} P<0.01$ ) (Figure 3A,B). Brain water content also markedly declined in the Tim- $3^{-1-}$ ICH group in all tested time points (the
Tim-3 $3^{-1-}$ ICH group versus the WT ICH group, $12 \mathrm{~h}$ $P<0.01$; $1 \mathrm{~d} P<0.01$; $3 \mathrm{~d} P<0.01$; $5 \mathrm{~d} P<0.01$; $7 \mathrm{~d} P<0.05$.) (Figure $3 C$ ). At 12 hours and day 1, there was no difference of NDS between the Tim- $3^{-/-} \mathrm{ICH}$ group and the WT ICH group. At 3 days, the NDS of the Tim $-3^{-/-} \mathrm{ICH}$ group was lower than that of the WT ICH group, but there was no statistical difference. At 5 and 7 days, there was a significant difference between two groups $(P<0.05)$ (Figure 3D), which meant that blockage of expression of Tim-3 could improve the neurological deficit after ICH.

The change of astrocytes, neutrophils and microglia/ macrophages after intracerebral hemorrhage

We investigated the number of astrocytes, neutrophils and microglia/macrophages in all groups using immunofluorescence staining. The results indicated that the number of 

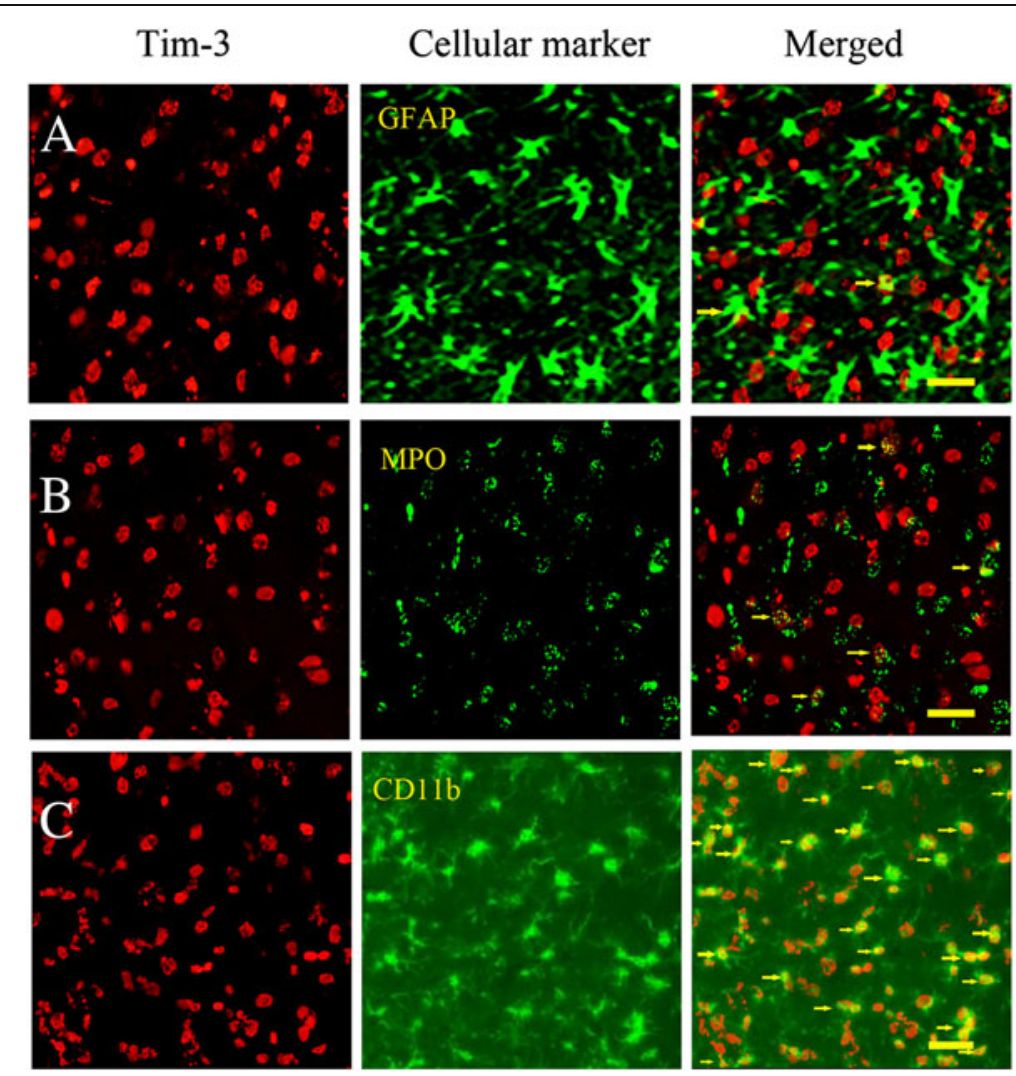

Figure 2 The expression of Tim-3 in different brain cells at day 1 after intracerebral hemorrhage (ICH). Double-immunofluorescence staining displayed that: A) A few of Tim-3 positive cells could express in GFAP ${ }^{+}$cells (astrocytes). B) A few of Tim-3 positive cells could express in $\mathrm{MPO}^{+}$cells (neutrophils), but more than were expressed in GFAP ${ }^{+}$cells (astrocytes). The arrows indicate co-expressed cells of Tim-3 and MPO. C) Almost all CD $11 b^{+}$cells (microglia/macrophages) were Tim-3 positive cells at day 1 after ICH. The arrows indicate co-expressed cells of Tim-3 and CD11b. Scale bar $=20 \mu \mathrm{m}$.

astrocytes, neutrophils and microglia/macrophages all increased after ICH. But the peak times of the three cells were different. The number of astrocytes elevated at Day 1, peaked at Day5 and Day 7. The number of neutrophils rose at 12 hours, peaked at Day 3, and then gradually declined. But the number of astrocytes and neutrophils were not different between the WT ICH group and the Tim- $3^{-/-}$ICH group. The number of microglia/macrophages increased at 12 hours and peaked at Day 1. This variation was the same as that of Tim-3, IL- $1 \beta$ and TNF-a. In Tim- $3^{-1-}$ ICH mice, the number of microglia/macrophages was obviously less than that of WT ICH mice and there was a significant difference between the two groups $(P<0.01)$ (Figure 5).

\section{Discussion}

In the present study we wanted to prove the effect of Tim-3 on brain inflammation after ICH. Three aspects of this research had to be addressed. The first aspect involved the expression of Tim-3 in the peri-hematomal zone after ICH. The second was related to the cellular resource of Tim-3 expression. The third was concerned with the correlation between the expression of Tim-3 and the severity of inflammatory response and brain edema. Furthermore, we examined the change in the release of inflammatory cytokines, brain edema, neurofunctional impairment and inflammatory cells (astrocytes, neutrophils and microglia/macrophages) in Tim- $3^{-/-} \mathrm{ICH}$ mice.

\section{The expression of Tim-3 in the peri-hematomal zone after} $\mathrm{ICH}$

Tim-3 is a new immuno-regulation molecule. It has been proved that Tim-3 extensively expresses itself in innate immune cells, including microglia, monocytes, mast cells, dendritic cells and natural killer cells, and plays complex roles in immune regulation and tolerance $[11,13,14,21-23]$. Tim-3 is involved in several inflammatory diseases, such as experimental autoimmune encephalomyelitis (EAE) [11], non-obese diabetes [24] and Coxsackievirus B3-induced myocarditis [21]. Anderson et al. [14] demonstrated that Tim-3 mRNA levels were much higher in inflamed white matter tissue in patients with multiple sclerosis (MS) and rat MS models, and 

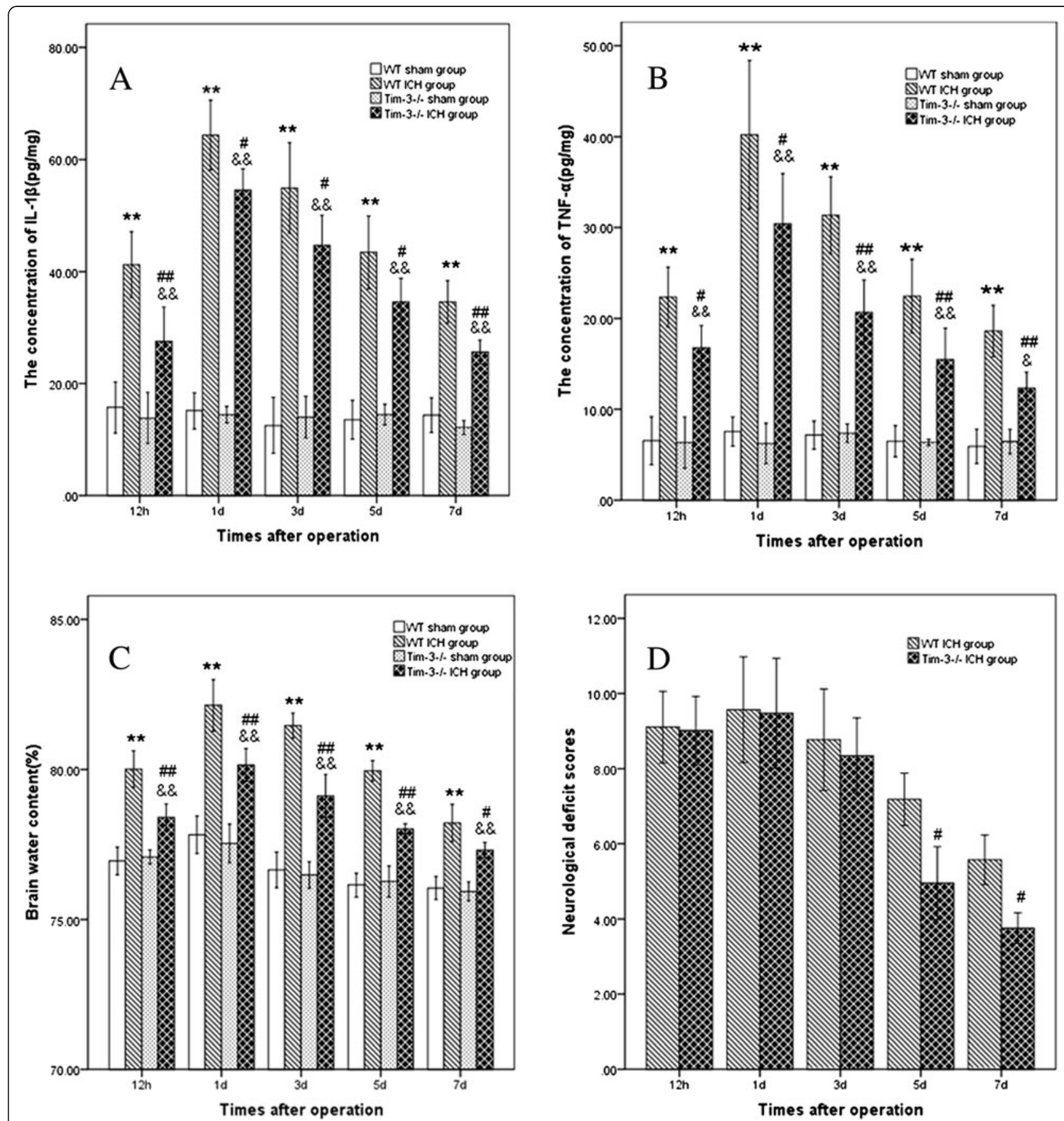

Figure 3 The change of IL-1 $\beta$, TNF-a, brain water content and neurological deficit scores (NDS) after intracerebral hemorrhage (ICH). A) indicates the change of the concentration of IL-1 $\beta$ in the peri-hematomal brain tissues at 12 hours andat 1, 3, 5 and 7 days post-ICH. B) shows the change of the concentration of TNF-a. C) displays the change of brain water content. D) shows the NDS between the Tim- $3^{-/-} \mathrm{ICH}$ group and the wild type (WT) ICH group. The WT ICH group $(n=6)$ versus the WT sham group $(n=6)$ : **P $<0.01$; The Tim- $3^{-1-}$ ICH group $(n=6)$ versus the Tim- $3^{-/-}$sham group $(n=6): \& \& P<0.01$; The Tim- $3^{-/-}$ICH group $(n=6)$ versus the WT ICH group $(n=6):{ }^{\prime} P<0.05,{ }^{\# \#} P<0.01$.

Tim-3 upregulated on $\mathrm{CD}_{11 \mathrm{~b}^{+}}$peripheral monocytes and resident microglia to promote TNF-a secretion. Zhao et al. [25] investigated the expression of Tim-3 in the acute phase of ischemic stroke, and indicated that overexpression of Tim-3 both in brain tissues of ischemiareperfusion mice and in peripheral blood mononuclear cells of patients with ischemic stroke positively correlated with plasma IL-17 and TNF- $\alpha$. All these researchers suggested that Tim-3 took parts in inflammatory-immunologic reaction. But it is completely unknown whether or not Tim-3 plays roles in ICH. Now, we have experimentally found that Tim-3 increases in the mouse 

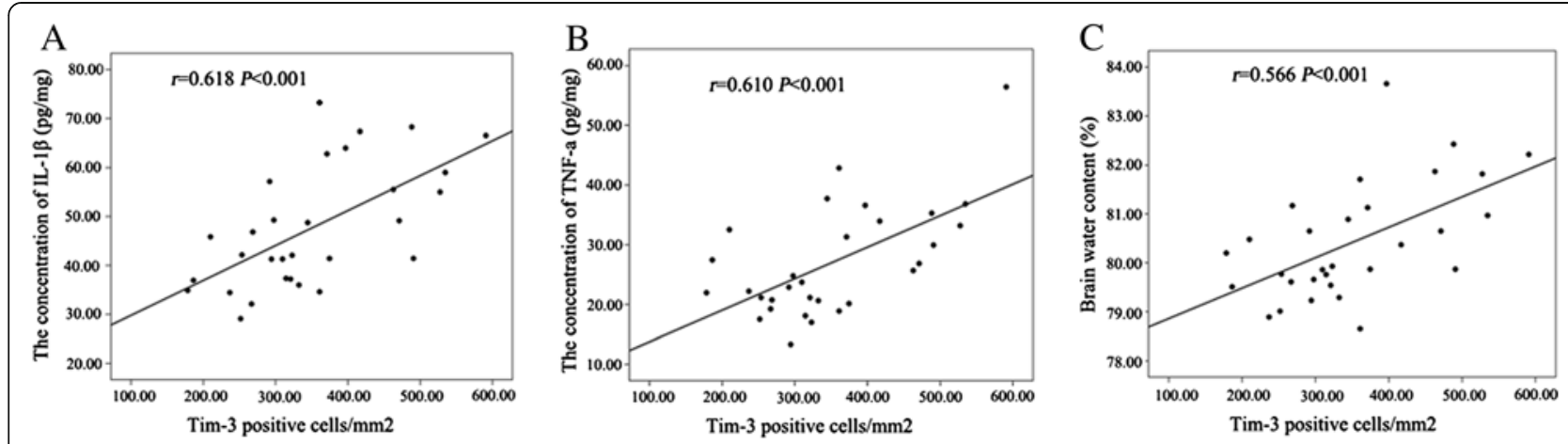

Figure $\mathbf{4}$ The correlation between the expression of Tim-3 and IL-1 $\boldsymbol{\beta}$, TNF-a, brain water content. A), B) and C) indicate that the expression of Tim-3 was positively correlated with $\mathrm{IL}-1 \beta(r=0.618, P<0.001)$, TNF-a $(r=0.610, P<0.001)$ and brain water content $(r=0.566, P=0.001)$.

peri-hematomal brain tissues early after autologous blood injection and progressively increasesand accompanies the development of brain inflammation, suggesting that Tim-3 is also involved in the inflammatory response following $\mathrm{ICH}$.

\section{The cellular resource of Tim-3 expression}

Previous studies have revealed that brain inflammation after ICH was characterized by the soakage of neutrophils and macrophages from blood and activation of microglia in brain tissues $[8,26]$. Among all inflammatory cells, microglia are the first non-neuronal cells to react to brain damage [27]. The shape of activated microglia is the same as the shape of blood-derived macrophages, so it is impossible to discriminate them from infiltrating macrophages. Microglia are activated at 1 hour after $\mathrm{ICH}$, much earlier than neutrophil infiltration, and the latter appears at 4 to $5 \mathrm{~h}$ after $\mathrm{ICH}$ [9]. Activated microglia/macrophages can release cytotoxic mediators, such as IL-1 $\beta$ and TNF-a, inducing brain inflammatory reaction and secondary damage $[10,15,28]$. Blockage of microglia activation with tuftsin fragment $1-3$ can attenuate neuroinflammation and brain damage after $\mathrm{ICH}$ in rats $[24,29]$. In this experimental study, we found that the expression of Tim-3 following ICH was preponderant in microglia/macrophages, was lower in neutrophils, and was lowest in astrocytes. Although astrocytes, neutrophils and microglia/macrophages all increased following $\mathrm{ICH}$, but only variation of microglia/macrophages was the same as that of Tim-3, IL-1 $\beta$ and TNF-a. These indicate that Tim-3 can inhibit the activation of microglia/macrophages, but not influence the astrocytes and neutrophils. Tim-3 not only expressed in microglia/macrophages, but also could regulate the function of them.
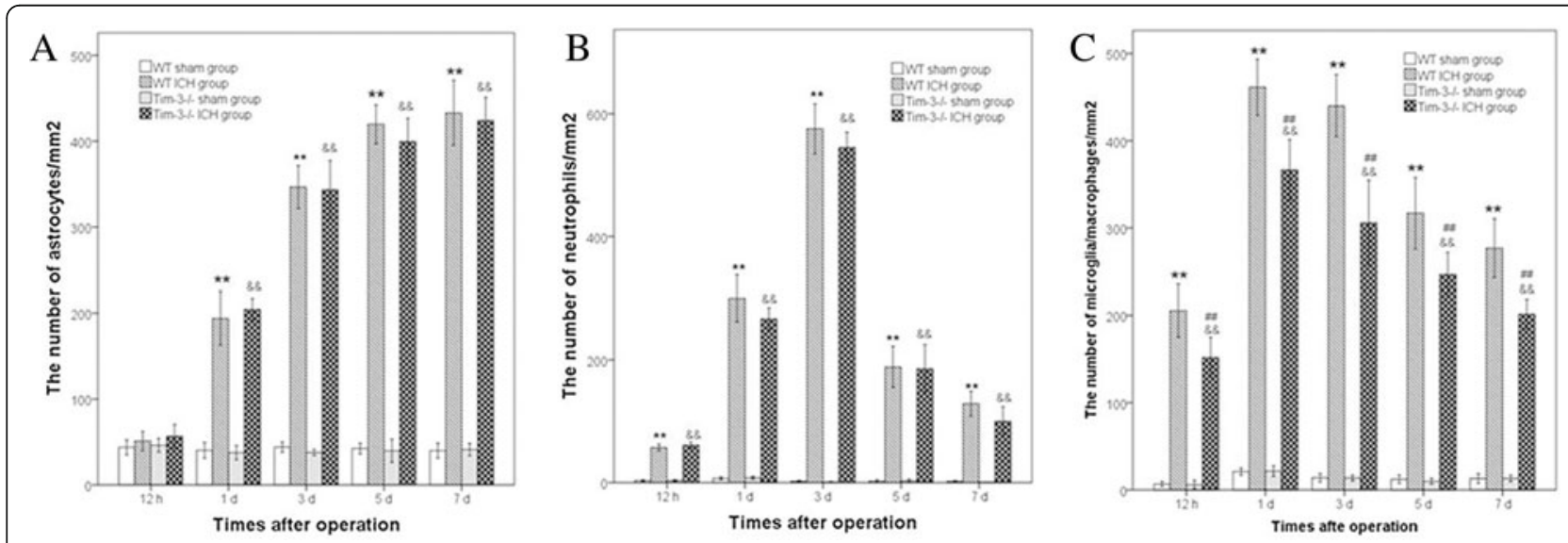

Figure 5 The change of the number of astrocytes, neutrophils and microglia/macrophages after intracerebral hemorrhage (ICH). A) indicates the change in the number of astrocytes in the peri-hematomal brain tissues at 12 hours and at 1, 3,5 and 7 days post- $\mathrm{ICH}$. B) shows the change in the number of neutrophils. $\mathbf{C})$ displays the change in the number of microglia/macrophages in the wild type (WT) ICH group $(n=6)$ versus the WT sham group $(n=6)$ : ${ }^{*} P<0.01$; The Tim- $-3^{-/-} I C H$ group $(n=4)$ versus the Tim- $3^{-/-}$sham group $(n=4)$ : \&\&P<0.01; the $\mathrm{Tim}^{-3^{-/-}} \mathrm{ICH}$ group $(\mathrm{n}=4)$ versus the WT ICH group $(\mathrm{n}=6)$ : ${ }^{\# \#} P<0.01$. 
The relationship between the expression of Tim-3 and the severity of inflammatory response and brain edema

IL-1 $\beta$ and TNF-a are two important proinflammatory cytokines, which can drive the inflammatory process and aggravate inflammation $[30,31]$. Although IL- $1 \beta$ and TNFa are released by many cells, including microglia/macrophages, astrocytes and neurons, the major source of these are activated microglia/macrophages [4,19,20]. Many experimental data have revealed that expression of IL-1 $\beta$ and TNF-a increase after ICH, and are associated with brain edema and brain damage. Increased expression of IL-1 $\beta$ and TNF-a can been detected not only in the central nervous system but also in systemic circulation [32-34]. IL-1 $\beta$ and TNF- $\alpha$ can boost inflammatory reaction in the early stage by promoting secretion of other chemotactic factors and adhesion molecules of the vascular endothelium, which can lead to the early infiltration of macrophages and neutrophils to the injury lesion $[35,36]$. The levels of TNF- $\alpha$ and IL-1 $\beta$ can represent the severity of brain inflammatory to a great degree. In our study, we found that TNF- $\alpha$ and IL- $1 \beta$ increased at 12 hours post$\mathrm{ICH}$, peaked at Day 1 and decreased at Day 3 after $\mathrm{ICH}$. The expression of Tim-3 is positively correlated to levels of TNF- $\alpha$, IL- $1 \beta$ and brain water content. Above all, we can deduce that augmented Tim-3 expression may promote inflammation and brain edema in the perihematomal tissues after $\mathrm{ICH}$ by regulating the function of microglia/macrophage. This result was supported by three points below. First of all, expression of Tim-3 increased early in the perilesional tissue and predominantly expressed itself in microglia/macrophages after ICH. The number of microglia/macrophages was much lower in Tim $-3^{-/-}$ICH mice. Furthermore, the secretion of TNF- $\alpha$ and IL-1 $\beta$ elevated in the peri-hematomal tissues following $\mathrm{ICH}$, and microglia/macrophages are the major types of cells to secrete TNF-a and IL-1 $\beta$. In addition, there was a positive correlation between the expression of Tim-3 and the levels of TNF- $\alpha$, IL- $1 \beta$ and brain water content. Blocking expression of Tim-3 could not only reduce the levels of TNF- $\alpha$, IL-1 $\beta$, brain water content and the number of microglia/macrophages, but also improve neurological function, which also elucidated the effect of Tim-3 on brain inflammatory reaction from another aspect. But the signal pathways of Tim-3 that regulate the function of microglia/macrophages need to be further explored.

\section{Conclusions}

Tim-3 plays an important role in the brain inflammation after ICH by regulating the function of microglia/macrophages and may be a potential treatment target.

\section{Abbreviations}

ELISA: Enzyme-linked immunosorbent assay; ICH: Intracerebral hemorrhage; MABP: Mean arterial blood pressure; NDS: Neurological deficit scores; RT-PCR: Reverse transcription polymerase chain reaction.
Competing interests

The authors declare that they have no competing interests.

\section{Authors' contributions}

The research was based on the original idea of CJX and YGL. CJX worked on the development of animal model, immunofluorescent staining, behavioral studies, and data analysis and drafted the manuscript. TW worked on the development of animal model, behavioral studies and real-time RT PCR. SC worked on the development of animal model, behavioral studies, ELISA and brain water content. YGL provided the equipment and wrote the manuscript. All authors read and approved the final manuscript.

\section{Acknowledgments}

The authors wish to thank Qian Jing for her technical assistance during the study as well as YangYang Cui for editorial assistance.

Received: 5 September 2013 Accepted: 17 November 2013

Published: 1 December 2013

\section{References}

1. Wang J, Doré S: Heme oxygenase 2 deficiency increases brain swelling and inflammation after intracerebral hemorrhage. Neurosci 2008, 155:1133-1141.

2. Qureshi Al, Mendelow AD, Hanley DF: Intracerebral haemorrhage. Lancet 2009, 373:1632-1644.

3. Wu G, Bao X, Xi G, Keep RF, Thompson BG, Hua Y: Brain injury after intracerebral hemorrhage in spontaneously hypertensive mice. J Neurosurg 2011, 114:1805-1811.

4. Wang J, Doré S: Inflammation after intracerebral hemorrhage. J Cereb Blood Flow Metab 2007, 27:894-908.

5. Wang J: Preclinical and clinical research on inflammation after intracerebral hemorrhage. Prog Neurobiol 2010, 92:463-477.

6. Aronowski J, Hall CE: New horizons for primary intracerebral hemorrhage treatment: experience from preclinical studies. Neuro Res 2005, 27:268-297.

7. Zhang D, Hu X, Qian L, Wilson B, Lee C, Flood P, Langenbach R, Hong JS: Prostaglandin E2 released from activated microglia enhances astrocyte proliferation in vitro. Toxicol Appl Pharmacol 2009, 238:64-70.

8. Wang J, Tsirka SE: Contribution of extracellular proteolysis and microglia to intracerebral hemorrhage. Neurocrit Care 2005, 3:77-85.

9. Wang J, Doré S: Heme oxygenase-1 exacerbates early brain injury after intracerebral haemorrhage. Brain 2007, 130:1643-1652.

10. Gao Z, Wang J, Thiex R, Rogove AD, Heppner FL, Tsirka SE: Microglial activation and intracerebral hemorrhage. Acta Neurochir Suppl 2008, 105:51-53.

11. Monney L, Sabatos CA, Gaglia JL, Ryu A, Waldner H, Chernova T, Manning S, Greenfield EA, Coyle AJ, Sobel RA, Freeman GJ, Kuchroo VK: Th1-specific cell surface protein Tim-3 regulates macrophage activation and severity of an autoimmune disease. Nature 2002, 415:536-541.

12. Sánchez-Fueyo A, Tian J, Picarella D, Domenig C, Zheng XX, Sabatos CA, Manlongat N, Bender O, Kamradt T, Kuchroo VK, Gutiérrez-Ramos JC, Coyle AJ, Strom TB: Tim-3 inhibits T helper type 1-mediated auto- and alloimmune responses and promotes immunological tolerance. Nat Immunol 2003, 4:1093-1101.

13. Nakae S, likura M, Suto $H$, Akiba $H$, Umetsu DT, Dekruyff $R H$, Saito $H$, Galli SJ: TIM-1 and TIM-3 enhancement of Th2 cytokine production by mast cells. Blood 2007, 110:2565-2568

14. Anderson AC, Anderson DE, Bregoli L, Hastings WD, Kassam N, Lei C, Chandwaskar R, Karman J, Su EW, Hirashima M, Bruce JN, Kane LP, Kuchroo VK, Hafler DA: Promotion of tissue inflammation by the immune receptor Tim-3 expressed on innate immune cells. Science 2007, 318:1141-1143.

15. Lin S, Yin Q, Zhong Q, Lv FL, Zhou Y, Li JQ, Wang JZ, Su BY, Yang QW: Heme activates TLR4-mediated inflammatory injury via MyD88/TRIF signaling pathway in intracerebral hemorrhage. J Neuroinflammation 2012, 9:46-60.

16. Gong C, Hoff JT, Keep RF: Acute inflammatory reaction following experimental intracerebral hemorrhage in rat. Brain Res 2000, 14:57-65.

17. Bond BC, Virley DJ, Cairns NJ, Hunter AJ, Moore GB, Moss SJ, Mudge AW, Walsh FS, Jazin E, Preece P: The quantification of gene expression in an animal model of brain ischaemia using TaqMan real-time RT-PCR. Brain Res 2002, 106:101-116. 
18. Xu X, Zhang J, Chen X, Liu J, Lu H, Yang P, Xiao X, Zhao L, Jiao Q, Zhao B, Zheng $P$, Liu Y: The increased expression of metabotropic glutamate receptor 5 in subventricular zone neural progenitor cells and enhanced neurogenesis in a rat model of intracerebral hemorrhage. Neurosci 2012, 27:474-483

19. Emsley HC, Tyrrell PJ: Inflammation and infection in clinical stroke. J Cereb Blood Flow Metab 2002, 22:1399-1419.

20. Fang $H$, Wang PF, Zhou Y, Wang YC, Yang QW: Toll-like receptor 4 signaling in intracerebral hemorrhage-induced inflammation and injury. J Neuroinflammation 2013, 17:10-27.

21. Frisancho-Kiss S, Nyland JF, Davis SE, Barrett MA, Gatewood SJ, Njoku DB, Cihakova D, Silbergeld EK, Rose NR, Fairweather D: Cutting edge: T cell Ig mucin-3 reduces inflammatory heart disease by increasing CTLA-4 during innate immunity. J Immunol 2006, 176:6411-6415.

22. Khademi M, Illés Z, Gielen AW, Marta M, Takazawa N, Baecher-Allan C, Brundin L, Hannerz J, Martin C, Harris RA, Hafler DA, Kuchroo VK, Olsson T, Piehl F, Wallström E: T Cell lg and mucin-domain-containing molecule-3 (TIM-3) and TIM-1 molecules are differentially expressed on human Th1 and Th2 cells and in cerebrospinal fluid-derived mononuclear cells in multiple sclerosis. J Immunol 2004, 172:7169-7176.

23. Wang F, He W, Zhou H, Yuan J, Wu K, Xu L, Chen ZK: The Tim-3 ligand galectin-9 negatively regulates CD8+ all reactive $T$ cell and prolongs survival of skin graft. Cell Immunol 2007, 250:68-74.

24. Anderson DE: TIM-3 as a therapeutic target in human inflammatory diseases. Expert OpinTher Targets 2007, 11:1005-1009.

25. Zhao D, Hou N, Cui M, Liu Y, Liang X, Zhuang X, Zhang Y, Zhang L, Yin D, Gao L, Zhang Y, Ma C: Increased T cell immunoglobulin and mucin domain 3 positively correlate with systemic IL-17 and TNF-a level in the acute phase of ischemic stroke. J Clin Immunol 2011, 31:719-727.

26. Wang J, Rogove AD, Tsirka AE, Tsirka SE: Protective role of tuftsin fragment $1-3$ in an animal model of intracerebral hemorrhage. Ann Neurol 2003 54:655-664.

27. Van Rossum D, Hanisch UK: Microglia. Metab Brain Dis 2004, 19:393-411.

28. Wu J, Yang S, Xi G, Song S, Fu G, Keep RF, Hua Y: Microglial activation and brain injury after intracerebral hemorrhage. Acta Neurochir Suppl 2008, 105:59-65.

29. Wang J, Tsirka SE: Tuftsin fragment $1-3$ is beneficial when delivered after the induction of intracerebral hemorrhage. Stroke 2005, 36:613-618.

30. Zheng Z, Yenari MA: Post-ischemic inflammation: molecular mechanisms and therapeutic implications. Neurol Res 2004, 26:884-892.

31. Kim JB, Choi J, Yu YM, Nam K, Piao CS, Lee MH, Han PL, Park JS, Lee JK: HMGB1, a novel cytokine-like mediator linking acute neuronal death and delayed neuroinflammation in the postischemic brain. J Neurosci 2006, 26:6413-6421

32. Allan SM, Rothwell NJ: Cytokines and acute neurodegeneration. Neurosci 2001, 2:734-744

33. Silva $Y$, Leira R, Tejada J: Molecular signatures of vascular injury are associated with early growth of intracerebral hemorrhage. Stroke 2005, 36:86-91.

34. Dziedzic T: Clinical significance of acute phase reaction in stroke patients. Front Biosci 2008, 13:2922-2927.

35. Barone FC, Feuerstein GZ: Inflammatory mediators and stroke: new opportunities for novel therapeutics. J Cereb Blood Flow Metab 1999, 19:819-834.

36. Stevens SL, Bao J, Hollis J, Lessov NS, Clark WM, Stenzel-Poore MP: The use of flow cytometry to evaluate temporal changes in inflammatory cells following focal cerebral ischemia in mice. Brain Res 2002, 932:110-119.

doi:10.1186/1742-2094-10-141

Cite this article as: Xu et al.: Increased expression of T cell immunoglobulin and mucin domain 3 aggravates brain inflammation via regulation of the function of microglia/macrophages after intracerebral hemorrhage in mice. Journal of Neuroinflammation 2013 10:141.

\section{Submit your next manuscript to BioMed Central and take full advantage of:}

- Convenient online submission

- Thorough peer review

- No space constraints or color figure charges

- Immediate publication on acceptance

- Inclusion in PubMed, CAS, Scopus and Google Scholar

- Research which is freely available for redistribution

Submit your manuscript at www.biomedcentral.com/submit 